\title{
Multi-Objective Artificial Bee Colony Algorithm to Estimate Transformer Equivalent Circuit Parameters
}

\author{
Züleyha YILMAZ ${ }^{1}$, Musab OKŞAR ${ }^{2}$, Fatih BAŞÇİFTÇí ${ }^{1}$ \\ ${ }^{1}$ Department of Computer Engineering,Selcuk University \\ 2 Electrical Engineer, Inonu University
}

\begin{tabular}{l} 
Article Info \\
\hline Article history: \\
Received May $23^{\text {th }}, 2017$ \\
Revised Aug $18^{\text {th }}, 2017$ \\
Accepted Oct $18^{\text {th }}, 2017$ \\
\hline Keyword: \\
Multi-objective artificial bee \\
colony algorithm \\
Multi-objective optimization \\
Transformer parameter \\
estimation
\end{tabular}

\section{Corresponding Author:}

Züleyha YILMAZ,

Department of Computer Engineering,

Selcuk University,

AlaeddinKeykubat Campus, 42075, Selcuklu, Konya, TURKEY.

Email: zuleyhayilmaz@selcuk.edu.tr

\section{Introduction}

Many engineering problems in the world have more than one objective. The main goal of the problems is to find optimum solutions for all objectives simultaneously. For this reason, the results are a set of optimum solution. In multi-objective problems (MOPs), problem solvers suggest this set of solutions to decision markers generally and decision makers decide an optimum solution from the set according to importance of the problem type. The set of optimum solutions is named as Pareto-optimal set [1]. Pareto-optimal set stores solutions which are not worse than each other. Namely, a Pareto-optimal solution is better than at least one of all objectives. These solutions are also named as non-dominated solutions [1]. This domination term is described as detailed in Section II. At this point, at the stage of proposing set of solutions, multi-objective optimization algorithms (MOAs) are utilized. In order to solve MOPs, proposed optimization algorithms are generally meta-heuristic algorithms. For instance, Multi-objective genetic algorithm is proposed to solve many MOPs such as flow shop scheduling [2] and fuzzy rule selection [3]. Multi-objective particle swarm optimization is also proposed to solve MOPs such as job-shop scheduling problem [4] and electromagnetic absorber design [5].

This study is focused on parameter estimation problem of a single-phase transformer being one of the engineering problems. Transformers are used as an electric machine to convert energy from one voltage to another voltage. In order to estimate transformer parameters, two experimental tests and some computations 
are required. This study provides parameters without any experimental test using proposed method with known current and voltage values at any known load.

So far, identification of the transformer parameter is realized as single optimization problem as [6], [7], [8]. However, in non-convex multi-objective optimization problems, results cannot be reached some optimum solutions when objectives of the problem are combined to do single objective problem [1]. Because of this reason, the transformer parameter estimation problem is solved as multi-objective optimization problem using Multi-Objective Artificial Bee Colony algorithm (MOABC) in this study. The algorithm is a population-based and stochastic optimization method. Foraging of real honey bees is simulated by the ABC. The original version of the $\mathrm{ABC}$ is for single optimization problems. Therefore, MOABC algorithm is utilized to identify transformer parameter being multi-objective problem.

In this paper, firstly described multi-objective optimization problems; then MOABC algorithm is described in detail; then introduced the problem and implementation of the MOABC to the problem. Finally, reported experimental results and conclusion.

\section{MaterialandMethods}

\subsection{Multi-ObjectiveOptimizationProblems}

MOPs involves more than one objective to find minimum or maximum solutions. In single objective optimization, there is sightful and only one purpose: to be minimized or maximized. On the contrary, in MOPs, there are conflicting objectives in some cases.

General form of MOPs can be expressed as (1), (2) and (3);

$$
\begin{gathered}
x=\left\{x_{1}, x_{2}, \ldots, x_{D}\right\} \\
\text { Minimize or Maximize } f(x)=\left\{f_{1}(x), f_{2}(x), \ldots, f_{m}(x)\right\} \\
\text { s.t. } g_{p}(x) \leq 0 ; p=1,2, \ldots, P \\
h_{n}(x)=0 ; n=1,2, \ldots, N
\end{gathered}
$$

where $x$ is a solution vector, $D$ is dimension of the problem. Also, $f(x)$ is called objective vector, $g$ and $h$ functions are constraints of the problem.

In MOPs, unlike single objective problems, the optimum solution is a solution set. Because, the solutions are trade-off and are known as Pareto-optimal set. This set refers to Pareto dominance [9], [10], [11]. The term of dominanceis frequently encountered term in MOPs. The states of dominance can be expressed by the following conditions [1];

If the solution $x_{1}$ dominates $x_{2}$, both case 1 and case 2 are verified;

Case 1: The objective values $\left(f_{1}\right.$ and $\left.f_{2}\right)$ are compared and the solution $x_{1}$ is found no worse than the solution $x_{2}$ for all objective.

Case 2: At least one objective, the solution $x_{1}$ outperforms the solution $x_{2}$.

Ifthesolution $x_{1}$ dominates all other solutions in the population, it is called non-dominated solution. Desired of MOPs, the solution set consists of non-dominated solutions. Representing of Pareto-optimal set andconcept of dominanceareillustrated as Fig. 1 fortwoobjectives $\left(f_{1}\right.$ and $\left.f_{2}\right)$ to be minimized [12]. 


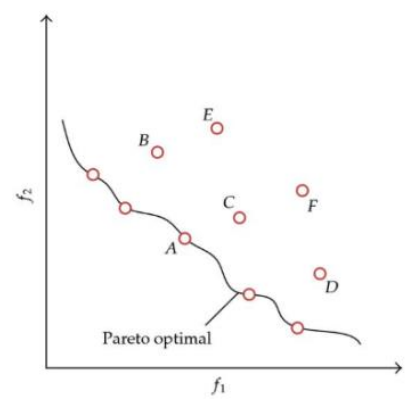

Figure 1. (a) Representing of Pareto-optimal set

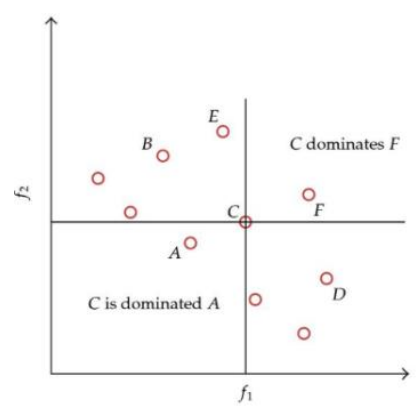

(b) Concept of Dominance

Tofind optimal set of MOPs, thealgorithmshavesomemechanisms. Twoimportantparts of themareExternal Archive and Update External Archive.

\subsubsection{External Archive (EA)}

In MOPs, despite of the fact that more than one objective, the algorithms generate an optimal solution set. The set maintains non-dominated solutions. To store these solutions, external archive can be used as [13], [14]. The archive is initialized in the first phase. Then, objective values of the solutions are compared and nondominated solutions are stored in the EA. Also, EA is updated in every cycle.

\subsubsection{Update External Archive}

In every cycle, the EA is updated. In this paper, updating procedure is associated with domination procedure. Every solution in population of the algorithm is respectively compared with the EA solutions. The solution in the EA dominated by population member is removed from the archive, and the dominant solution of the population is added into the EA. Not only dominant solutions are added, but also non-dominated solutions in the population are joined into the EA.

\subsection{Multi-ObjectiveArtificialBeeColonyAlgorithm}

The basic artificial bee colony algorithm (ABC) has been proposed by Karaboga [15] in 2005. ABC is a nature-spired algorithm. In nature, honey bees search food sources and when they find any source, they share the knowledge about the source with the other bees by dancing. Inspired by this communication, ABC algorithm is developed. Employed, onlooker and scout bees are the types of artificial bee in the algorithm. Also, initially, the initial parameters of colony size, maximum cycle and limit value are set. Limit value is the abandonment value of the food source. Initial population is generated by randomly and other phases are follows;

Employed bees: This type of bees calculates nectar amounts of the neighbor food sources. After comparing existing source and neighbor source, employed bees select the better source.

Onlooker bees: Using the information about the quality of food sources, onlooker bees interpret the sources. Then, all onlooker bees choose a food source and are fed.

Scout bees: In the ABC, employed bee turns into a scout bee when the food source is consumed (reaches the limit value).

The basic form of the algorithm is introduced for single optimization problems. In this study, the optimization problem has three objectives to be minimized. Therefore, the focus of the study is multi-objective ABC (MOABC) algorithm.

In MOABC, defining initial values of the food sources are the same with the basic ABC. It can be showed as (4);

$$
x_{i j}=x_{j}^{\text {min }}+\operatorname{rand}(0,1)\left(x_{j}^{\max }-x_{j}^{\text {min }}\right)
$$

$X_{i j}$ is $j$. dimension of $i$. food source where $i=(1, \ldots, F N($ Food Number $))$ and $j=(1, \ldots, D$ (Dimension)). The maximum and minimum bounds of the dimension is respectively $x_{j}^{\max }, x_{j}^{\min }$. 
One of the most important modification in MOABC is to determine neighbor food source. In the algorithm, each employed bee, which performs this part, utilizes EA member specified randomly to optimize its solution. It can be defined the following equation (5);

$$
v_{i j}=x_{i j}+\phi_{i j}\left(x_{i j}-\operatorname{ExAr}_{k j}\right)
$$

$V$ is neighbor solution of $X_{i}$ and $j$ is the random parameter of $X$ food source, and $k$ is the random member of the EA. $\phi_{i j}$ is a value between [-1,1]. Whenever the neighbor solution is determined, space bound is controlled. If a parameter exceeds the bounds, the parameter pulls the limits.

After determining the parameters, fitness values for all objectives of the two solutions are compared. This process uses greedy selection mechanism. Namely, the solution dominating the other solution is selected. If the selected solution is neighbor solution, then the abandonment value of the source is reset. Vice versa, abandonment value of the source is incremented. Fitness value is determined as (6);

$$
\text { fitness }_{i}= \begin{cases}1 /\left(1+f_{i}\right) & , f_{i} \geq 0 \\ 1+a b s\left(f_{i}\right) & , f_{i}<0\end{cases}
$$

fitness $_{i}$ is fitness value of $i$. food source for minimization problems. If the problem is maximization, $f_{i}$ denotes the fitness value.

After employed bees, new tasks are realized by onlooker bees. The mission is to choose a food source to optimize the solution using tournament selection for each onlooker bee. In tournament selection, two random food source is chosen from the population and onlooker bee choses the solution has better fitness value, and the neighbor food source is determined as employed bee part. Then, greedy selection is applied in this part, too. Abandonment value is updated according to which source is selected.

The last type of bee is scout bee. Scout bee part is completely as basic ABC. If a food source reaching the limit value, then the food source is exhausted. The parameters of the food source are randomly determined again and abandonment value is reset.

After completing all bee parts, updating EA procedure occurs. Each bee in the population is compared with the EA members according to fitness values. If the solution $x_{i}$ dominates the member of EA, the EA member is removed and the solution $x_{i}$ is inserted into the EA. If the solution $x_{i}$ and EA member are non-dominated solutions, then the solution $x_{i}$ is inserted into the EA.

Pseudocode of MOABC algorithm can be expressed as Fig. 2.

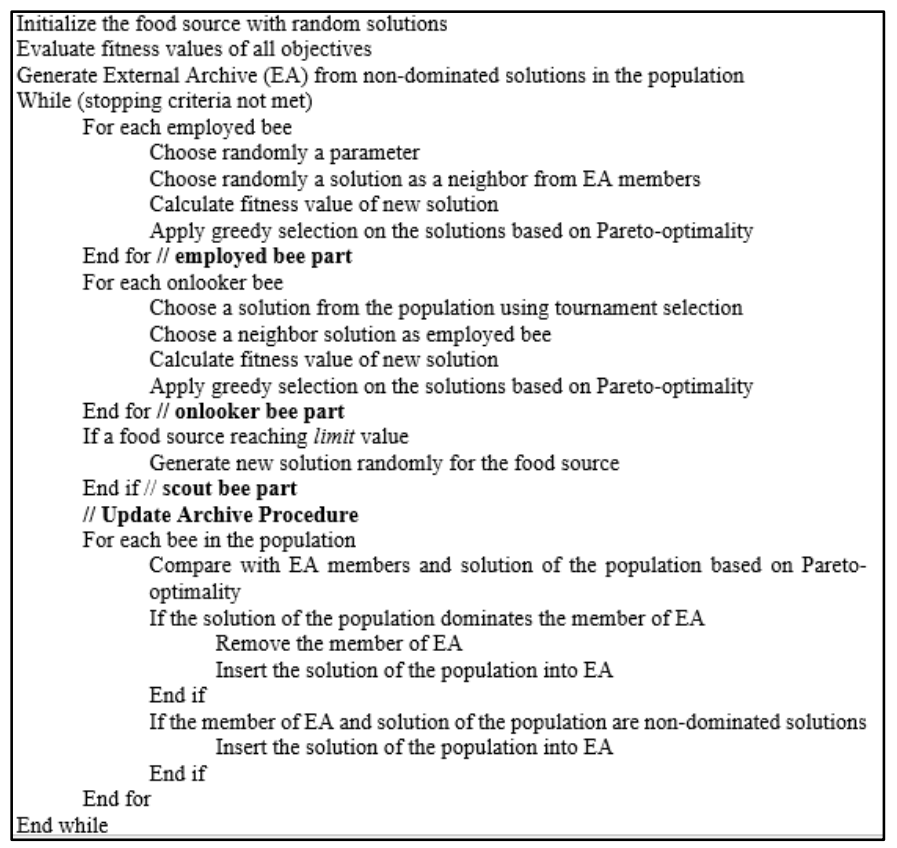

Figure 2. Pseudocode of the MOABC algorithm 


\section{TransformerEquivalentCircuitAndParameterEstimation Using MOABC}

Transmission of electricalpowerfromtheplace of productiontootherregions is providedbyalternativecurrent. Duetothehighvoltage of thealternatingcurrentelectricalpower, transformersareused. A transformerprovidestotransformfromthehighvoltage of electricalpowertolowvoltageorviceversa. Whiledoingthistransformation, nochange in thefrequency. A transformerstructureconsists of twowindingbeingcalledprimaryandsecondarywinding. Thetransformers can be classified as singlephaseandpoly-phasetransformers. Insingle-phasetransformers, primarywinding is forinputsignalwithsinglephase, andsecondarywinding can be oneormoreaccordingtoaim. On theotherhand, in poly-phasetransformers, number of primarywinding is twoormore, andsecondarywinding can be oneormoreaccordingtoaim. Poly-phasetransformersareoftenwrapped in threephases, andalsothisinformation can be found in detail in [16].

Inthisstudy, single-phasetransformersarefocuses on andtheequivalentcircuit of a single-phasetransformer is illustrated as Fig. 3. Whenestimation of thetransformerparameters is examined, twotestsarerequired: noloadandshortcircuitoperation. Thesetestsareexamined in detail in [16]. Owingtothisstudy, onlyfromcurrentandvoltage at anyknownload, thetransformerparametersareobtainedwithoutany test. Eachequation of transformerparameters is obtainedfromKirchhoff'scircuitlawsandtherebythreeobjectives of the problem areidentified.

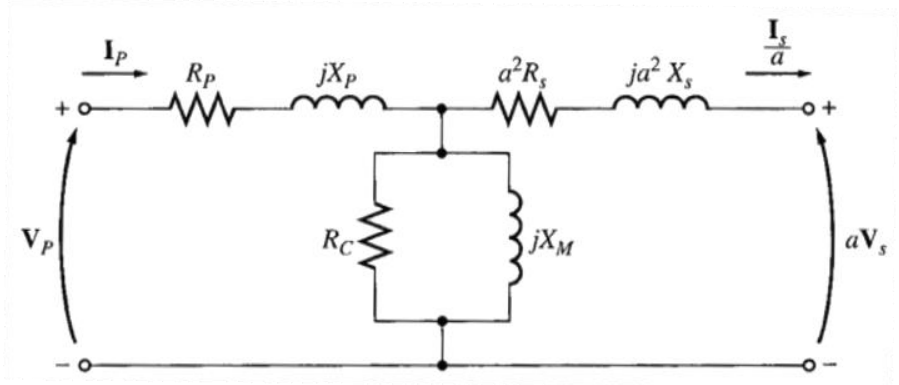

Figure 3. Equivalenttransformercircuitreferringtotheprimary

where $R_{p}$ is primarywindingresistance, $X_{p}$ is primarywindingleakagereactance, $V_{p}$ is primaryvoltage, $I_{p}$ is primarycurrent, $\quad a$ is transformingratio, $R_{s}$ is secondarywindingresistance, $X_{s}$ is secondarywindingleakagereactance, $I_{s}$ issecondarycurrent, $V_{s}$ is secondaryvoltage, $R_{c}$ is resistance of coreand $\mathrm{X}_{\mathrm{m}}$ is magnetizingreactance in theFig. 2.

$$
\begin{aligned}
& E r r_{1}=|| I_{p}\left|-A c t_{I p}\right| \\
& E r r_{2}=|| I_{s}\left|-A c t_{I S}\right| \\
& E r r_{3}=|| V_{S}\left|-A c t_{V S}\right|
\end{aligned}
$$

where $\operatorname{Err}_{1}$ is theerror of the $I_{p}, E_{2}$ is theerror of the $I_{s}{ }^{\prime}\left(I_{s} / a\right), E_{3}$ is theerror of theV $V_{s}{ }^{\prime}\left(a V_{s}\right) . \quad I_{p}, I_{s}$ and $\mathrm{V}_{\mathrm{s}}$ areobtainedbyKirchhoffCircuitLaws.

Inthisstage, MOABC createsrandomlyinitialparameterswhich of $R_{p}, \quad X_{p}, \quad R_{s}{ }^{\prime}\left(a^{2} R_{s}\right), \quad X_{s}{ }^{\prime}\left(a^{2} X_{s}\right)$, $\mathrm{R}_{\mathrm{c}}$ and $\mathrm{X}_{\mathrm{m}}$ andcalculatesfitnessvalues of theobjectivesbenefitingfromtheparameters. Then, nondominatedsolutionsarestored in the EA. Whilestoppingcriteria is not met, eachbeetriestoimprovethesolutions. Whenreachingstoopingcriteria, MOABC determinesPareto-optimal solutions of the problem in thearchive. AccordingtotheEuclidiandistancemethod, thebestsolution is selectedthroughthe EA members. Finding minimum solution in the EA is associatedwithparameters of the problem.

\section{ExperimentalResults}

MOABC is used to estimate transformer parameters according to current and voltage at any known load in this study. The performance of the MOABC is investigated in two different load.

In Case I, the transformer parameters $\left(\mathrm{R}_{\mathrm{p}}, \mathrm{X}_{\mathrm{p}}, \mathrm{R}_{\mathrm{s}}{ }^{\prime}, \mathrm{X}_{\mathrm{s}}{ }^{\prime}, \mathrm{R}_{\mathrm{c}}\right.$ and $\left.\mathrm{X}_{\mathrm{m}}\right)$ are determined using MOABC at a $\mathrm{Z}_{\mathrm{L} 1}$ load $\left(Z_{\mathrm{Ll}}=50+\mathrm{i} 30 \Omega\right)$. Actual data can be obtained by experimental tests which are no-load and short-circuit operation. This study assumes that the actual parameters are already known. Then, current and voltage $\left(\mathrm{I}_{\mathrm{p}}\right.$, $\mathrm{I}_{\mathrm{s}}$ ' and $\left.\mathrm{V}_{\mathrm{s}}{ }^{\prime}\right)$ are calculated according to estimated parameters. Actual data, estimated data and error rate are 
showed in Table I. The results show that proposed method is very satisfied for estimation parameters of the single-phase transformer.

This study is for estimation of the parameters even if at different load operations. Therefore, the results are demonstrated at the another $\mathrm{Z}_{\mathrm{L} 2}$ load $\left(\mathrm{Z}_{\mathrm{L} 2}=22.3607 \Omega\right)$ in Case II as Table II.The results show again superior performance of MOABC algorithm to estimate same parameters at another load.

Table 1. Case I: EstimatedParameters of theSingle-PhaseTransformerobtainedby MOABC algorithm at $\mathrm{Z}_{\mathrm{L} 1}$ load.

\begin{tabular}{cccc}
\hline $\begin{array}{c}\text { Parameters and } \\
\text { Transformer Data }\end{array}$ & Actual Data & $\begin{array}{c}\text { Estimated Data by } \\
\text { MOABC }\end{array}$ & Error $(\%)$ \\
\hline $\operatorname{Rp}(\Omega)$ & 1 & 1.054 & -5.4 \\
$\operatorname{Xp}(\Omega)$ & 2 & 1.978 & 1.1 \\
$\operatorname{Rs}(\Omega)$ & 1.4 & 1.433 & -2.36 \\
$\operatorname{Xs}(\Omega)$ & 1.8 & 1.870 & -3.89 \\
$\operatorname{Rc}(\Omega)$ & 6400 & 6339.49 & 0.95 \\
$\operatorname{Xm}(\Omega)$ & 1100 & 1092.50 & 0.68 \\
$\operatorname{Ip}(A)$ & 3.65 & 3.6500 & 0 \\
Is' $(A)$ & 3.50 & 3.6279 & -3.65 \\
$\operatorname{Vs}^{\prime}(\mathrm{V})$ & 205.37 & 211.42 & -2.95 \\
\hline
\end{tabular}

Table 2.Case II: EstimatedParametersobtainedby MOABC at $\mathrm{Z}_{\mathrm{L} 2} \mathrm{load}$.

\begin{tabular}{cccc}
\hline $\begin{array}{c}\text { Parameters and } \\
\text { Transformer Data }\end{array}$ & Actual Data & $\begin{array}{c}\text { Estimated Data } \\
\text { by MOABC }\end{array}$ & Error $(\%)$ \\
\hline $\operatorname{Rp}(\Omega)$ & 1 & 1.2320 & -23.2 \\
$\operatorname{Xp}(\Omega)$ & 2 & 2.1693 & -8.47 \\
$\operatorname{Rs}^{\prime}(\Omega)$ & 1.4 & 1.1617 & 17.02 \\
$\operatorname{Xs}^{\prime}(\Omega)$ & 1.8 & 1.6326 & 9.3 \\
$\operatorname{Rc}(\Omega)$ & 6400 & 6442.27 & -0.66 \\
$\operatorname{Xm}(\Omega)$ & 1100 & 1088.43 & 1.05 \\
$\operatorname{Ip}(\mathrm{A})$ & 8.47 & 8.4648 & 0.06 \\
Is' $(\mathrm{A})$ & 8.45 & 8.4514 & -0.02 \\
Vs' $(\mathrm{V})$ & 202.27 & 202.33 & -0.03 \\
\hline
\end{tabular}

\section{Conclusion}

This paper is focused on MOABC algorithm to estimate single-phase transformer parameters via current and voltage values at any known load. ABC algorithm simulates honey bee behavior. The reason for choosing this algorithm is simple implementation and minimum control parameters. In optimization process, the algorithm tries to minimize error between actual and estimated data. Proposed MOABC uses Pareto-optimality concept to achieve optimum solutions of the problem. Through the non-dominated solutions, one optimum solution is selected using Euclidian distance method. The results of the proposed method show that using MOABC algorithm to estimate transformer equivalent circuit parameters very satisfactory results is obtained.

\section{Acknowledgements}

This study is supported by Academic Stuff Training Program of Selcuk University, Konya, Turkey. The Project number is 2015-ÖYP-005. 


\section{References}

[1]. K. Deb, Multi-Objective optimization using evolutionary algorithms, John Wiley \& Sons, Inc. New York, NY, USA, 2001.

[2]. T. Murata, H. Ishibuchi, and H. Tanaka, "Multi-objective genetic algorithm and its applications to flowshop scheduling," Computers \& Industrial Engineering, vol. 30, pp. 957-968, 1996.

[3]. H. Ishibuchi and T. Yamamoto, "Fuzzy rule selection by multi-objective genetic local search algorithms and rule evaluation measures in data mining," Fuzzy sets and systems, vol. 141(1), pp. 59-88, 2004.

[4]. G. Zhang, X. Shao, P. Li, and L. Gao, "An effective hybrid particle swarm optimization algorithm for multi-objective flexible job-shop scheduling problem," Computers \& Industrial Engineering, vol. 56 (4), pp. 1309-1318, 2009.

[5]. S. Chamaani, S. A. Mirtaheri, M. Teshnehlab, and M. A. Shooredeli, "Modified multi-objective particle swarm optimization for electromagnetic absorber design," In Applied Electromagnetics, 2007. APACE 2007. Asia-Pacific Conference on IEEE, pp. 1-5, December, 2007.

[6]. D. Meister and M. A. G. de Oliveira, "The use of the least squares method to estimate the model parameters of a transformer," 2009 10th International Conference on Electrical Power Quality and Utilisation, Lodz, doi: 10.1109/EPQU.2009.5318853, pp. 1-6, 2009.

[7]. S. A. Soliman, R. A. Alammari, and M. A. Mostafa, "On-line estimation of transformer model parameters," 2004 Large Engineering Systems Conference on Power Engineering (IEEE Cat. No.04EX819), doi: 10.1109/LESCPE.2004.1356295, pp. 170-178, 2004.

[8]. S. H. Thilagar and G. S. Rao, "Parameter estimation of three-winding transformers using genetic algorithm," Eng. Appl. Artificial Intell., vol. 15, no. 5, pp. 429-437, Sep. 2002.

[9].K. Deb, "A fast and elitist multiobjective genetic algorithm: NSGA-II", IEEE Transactions On Evolutionary Computation, vol. 6, pp. 182-197, 2002.

[10].A. H. F. Dias and J. A. de Vasconcelos, "Multiobjective genetic algorithms applied to solved optimization problems," IEEE Transactions On Magnetics, vol. 38, no. 2, pp. 1133-1136, Mar. 2002.

[11].A. Osyczka. "Evolutionary algorithms for single and multicriteria design optimization," New York: Physica Verlag., 2002.

[12].W. Zou, Y. Zhu, H. Chen, and B. Zhang, "Solving multiobjective optimization problems using artificial bee colony algorithm," Discrete Dynamics in Nature and Society, vol. 2011, 37 pages, 2011.

[13].J. D. Knowles and D. W. Corne, "Approximating the nondominated front using the pareto archived evolution strategy," Evolutionary Computation, vol. 8(2), pp. 149-172, 2000.

[14].C. A. Coello and G. T. Pulido, "A micro-genetic algorithm for multiobjective optimization," in Proc. EMO 2001, pp. 126-140, Mar. 2001.

[15].D. Karaboga, An Idea Based On Honey Bee Swarm for Numerical Optimization, Technical Report TR06, Erciyes University, Engineering Faculty, Computer Engineering Department, 2005.

[16].S. J. Chapman, Electric Machinery Fundamentals, McGraw-Hill, New York, 2003. 